\title{
Swarm Intelligence Algorithms as the Promising Tool for Solving Optimisation Problems
}

\author{
Nafrizuan Mat Yahya* \\ Faculty of Manufacturing Engineering, University Malaysia Pahang, Pahang, Malaysia
}

Submission: July 19, 2017; Published: September 22, 2017

*Corresponding author: Nafrizuan Mat Yahya, Faculty of Manufacturing Engineering, University Malaysia Pahang, Pahang, Malaysia, Tel: +6018-6652051, Email: nafrizuanmy@ump.edu.my

Keywords: Genetic algorithm (GA); Evolutionary strategy (ES); Evolutionary programming (EP); Genetic programming (GP); Differential evolution (DE); Bacterial foraging optimisation (BFO); Artificial bee colony (ABC); Particle swarm optimisation (PSO); Ant colony optimization (ACO); Artificial immune system (AIS); Bat Algorithm (BA)

\section{Introduction}

A quote by George Bernhard Dantzig, a famous American mathematical scientist who made important contributions to operations research, computer science, economics, and statistics [1]: "True optimisation is the revolutionary contribution of modern research to decision processes". Optimisation according to the definition of Merriam-Webster Dictionary Merriam-Webster [2] is an act, process, or methodology of making something (as a design, system, or decision) as fully perfect, functional, or effective as possible.

In general, optimisation is the process of obtaining either the best minimum or maximum result under specific circumstance Singiresu [3], Xin-She \& Suash [4]. Sanghamitra \& Sriparna [5], Statnikov et al. [6], Xin-She [7] added that the optimisation process engages with defining and examining objective or fitness function that suits some parameters and constraints. Nowadays, a vast range of business, management and engineering applications utilise the optimisation approach to save time, cost and resources while gaining better profit, output, performance and eficiency.

Optimisation problems can be divided into two categories: continuous and combinatorial (discrete) Laszlo [8]. A combinatorial optimisation problem has a finite number of solutions but this is not in the case with a continuous optimisation problem where the number of solutions is infinite. This research concentrates only on continuous optimisation problems. So in this thesis, optimisation will refer solely to continuous optimisation problems.

Normally, the optimisation problems can further be classified into two major types namely; single objective optimisation and multi objective optimisation. Naturally, solving a single objective optimisation is about finding an optimised solution to the problem at hand based on the single objective. Multi objective optimisation, on the other hand, is multifaceted and solving the problem is to seek compromised solutions based on a set of conflicting objectives Castro-Gutierrez et al. [9], Dragan \& Parmee [10], Ivan [11], Xin-She [12]. As there will be no unique solution to a multi objective optimisation problem Ngatchou et al. [13], a set of 'trade-o' solutions, referred to as Pareto optimum solutions, compromising the objectives is produced Coello [14], Zhou et al. [15].

Meanwhile, the single objective optimisation can be designated as either unconstrained or constrained depending on whether or not the problem contains constraints. Conn et al. [16] elaborates the unconstrained single objective optimisation problem (or widely known as single objective optimisation problem) as a problem that has no constraints specified on the variables and usually is less complicated. However, a constrained single objective optimisation problem (or widely referred as constrained optimisation problem) comes with lack of explicit mathematical formulation but has discrete definition domains, mixed with continuous and discrete design variables and also strong nonlinear objective functions with multiple complex constraints Leticia [17], Harish [18], Fei et al.[19].

According to Seok \& Zong [20], over the past forty years, many techniques have been established to solve different optimisation problems efficiently. On the words of Jones et al. [21]; many optimisation problems work with mathematical or 
numerical linear and nonlinear programming methods and use simple and ideal models to get the optimum result. However, stressed that the numerical optimisation method tends to improve the solution locally which is different from a real world problem, often more complex and unpredictable. In addition, due to their computational drawbacks, plus the requirement of substantial gradient information, traditional numerical programming strategies have been incapable of solving any optimisation problem consistently Sadollah et al. [22].

Due to stated limitations and other downsides as listed by Coello [14], the alternative prospect to solve an optimisation problem is by heuristic or metaheuristic method Liqun et al. [23], Tsung-Jung [24], Jacqueline \& Richard [25]. Even though the metaheuristic methods are computationally laborious and give no guarantee of the quality of the results as stated by XinShe [7], the methods are still in the top ranking of optimisation solving tools. Metaheuristic methods offer significant advantages such as; easy to develop and implement, with a broad range of applicability, able to give a global perspective to the problem domains that are needed to be solved Afshar et al. [26] and the convergence rate of the global or nearly global optimum results are better than other optimisation approaches Ali [27].

For the past decades, evolutionary algorithms that are part of metaheuristic methods have become popular among the researchers to deal with the complexity of a wide variety of single and multi objective optimisation problems Wenyin et al. [28], Yong et al. [29], Xin-She \& Gandomi [30]. Evolutionary algorithms have been derived from a combination of a set of rules or restrictions and randomness by populations in generations. Evolutionary algorithms imitate or simulate the successful characteristics of natural phenomena of physical systems (e.g. simulated annealing algorithm) or biological systems (e.g. animal behaviours-based algorithms) Ricardo \& Coello [31].

Evolutionary algorithms offer some advantages. According to Alex et al. [32], the major advantages of evolutionary algorithms are that they are very good in general applicability that cover the vast range of problems as well as prior knowledge of the problem considered as inessential. An evolutionary algorithms only needs an explicit or implicit objective function to optimise the problem Janez et al. [33], Qie \& Ling [34]. An evolutionary algorithm kicks off with some guessed solutions, updates solutions in a synergistic manner then navigates the search agents to balance between exploitation of good foundso-far positions and exploration of new anonymous search positions toward the optimum global solution Janez et al. [33], Qie H \& Ling [34], Hui et al. [35], Mezura-Montes E \& Coello [36], Zhang et al. [37]. Alex et al. [32] divided the evolutionary algorithms to some sub-fields. The sub fields include Genetic Algorithm (GA) by Holland in 1975, Evolutionary Strategy (ES) by Rechenberg in 1965, Evolutionary Programming (EP) by
Fogel in 1966, Genetic Programming (GP) by Koza in 1992 and Differential evolution (DE) by Storn and Price in 1995.

Among most popular evolutionary algorithms that have already captured the attention of researchers today are swarm intelligence algorithms. Swarm intelligence algorithms are inspired by the collective behaviour of swarms through a complex interaction between individuals and their neighbourhood with nature such as a colony of ants, bacteria, bees, bats, birds and fishes Leandro \& Viviana [38], Erik \& Miguel [39], Adil et al. [40]. In general, swarms have selforganisation and decentralised control features and all the swarm follows the same system where a population of swarm cooperates and interacts with each other in the group and the environment under certain rules during foraging or socialising. The most remarkable features of any swarm intelligence algorithm are that it has advantages of memory, diverse multicharacters capability, rapid solution improvement mechanism and is adaptable to internal and external changes Erik \& Miguel [39], Harish [18].

There are some well-known swarm intelligence algorithms developed over the past two decades. Kennedy \& Eberhart [41] pioneered Particle Swarm Optimisation (PSO) algorithm that simulates the social behaviour and choreography of a bird flock. It was followed by Ant Colony Optimisation (ACO) algorithm by Marco [42]. The algorithm simulates the activity of ants while seeking a path to a food source. In micron scale of swarm intelligence algorithms, the characteristics and behaviour of the vertebrate immune system have led Hofmeyr \& Forrest [43] to develop an Artificial Immune System (AIS) algorithm. Passino [44] successfully imitated the social foraging behaviour of Escherichia coli (E. -coli) for search of nutrients with the Bacterial Foraging Optimisation (BFO) algorithm.

In 2007, the Artificial Bee Colony (ABC) optimisation method that was modelled from a colony of bee raised attention of research community after developed by Dervis \& Bahriye [45]. Then, Timothy et al. [46] initiated Roach Infestation Optimisation (RIO) algorithm that was inspired from social characteristics of an intrusion of cockroaches. Later, XinShe [47] introduced Bat Algorithm (BA) which imitated the echolocation of bats to find prey with different levels of pulse and loudness emitted. The algorithm was the third from him after a Cuckoo Search (CS) algorithm Xin-She \& Suash [48] encouraged from compellation of social parasitism practised by a group of cuckoo and the firefly algorithm (FA) Xin-She [49] idealised from the flashing behaviour of fireflies a year before.

Tawfeeq [50] also utilised the concept of echolocation of bats to find prey to develop a new swarm intelligence algorithm. Different from the algorithm developed by Xin-She [47] as cited before, this algorithm models the principles of bats sonar used in echolocation to search for the optimum solution to a speci c 


\section{Robotics \& Automation Engineering Journal}

problem Tawfeeq [50]. It is worth mentioning, to strengthen the swarm intelligence algorithms or to cater for a specific problem, the versions of swarm intelligence algorithms hybridised between each other or with other conventional approaches have also existed.

\section{References}

1. Eberhard Z (1995) Applied functional analysis.

2. Merriam-Webster (2015) Words fail us. Merriam-webster dictionary, USA.

3. Singiresu SR (2009) Engineering optimization: theory and practice. ( $4^{\text {th }}$ edn), John Wiley \& Sons, New jercy, USA.

4. Xin-She Y, Suash D (2014) Cuckoo search: recent advances and applications. Neural Computing and Applications 24(1): 169-174.

5. Sanghamitra B, Sriparna S (2013) Some single-and multiobjective optimization techniques. Unsupervised Classification, p. 17-58.

6. Roman Statnikov, Josef Matusov, Alexander Statnikov (2012) Multicriteria engineering optimization problems: statement, solution and applications. Journal of Optimization Theory and Applications 155(2): 355-375.

7. Xin-She Y (2005) Engineering optimizations via nature-inspired virtual bee algorithms. A Bioinspired Approach, pp. 317-323.

8. Laszlo L (2010) Discrete and Continuous: Two sides of the same? In Visions in Mathematics, p.359-382.

9. Castro-Gutierrez J, Landa-Silva D, Moreno JP (2010) Improved dynamic lexicographic ordering for multi-objective optimisation. Parallel Problem Solving from Nature PPSN XI 6233: 31-40.

10. Dragan C, Parmee IC (1998) Evolutionary design and multi-objective optimisation. Intelligent Techniques and Soft Computing (EUFIT) Sixth European Congress, Aachen, Germany, pp. 397-401.

11. Ivan PS (2012) Compendious lexicographic method for multi-objective optimization. Ser Math Inform 27(1): 55-66.

12. Xin-She Y (2011) Bat algorithm for multi-objective optimisation. International Journal of Bio-Inspired Computation 3(5): 267-274.

13. Ngatchou P, A Zarei, A El-Sharkawi (2005) Pareto multi objective optimization. Proceedings of the $13^{\text {th }}$ International Conference, Virginia, USA, p. 84-91.

14. Coello CA (2006) Evolutionary multi-objective optimization: a historical view of the field. IEEE Computational Intelligence Magazine 1(1): 28-36

15. Zhou A, Qu BY, Li H, Zhao SZ, Suganthan PN, et al. (2011) Multiobjective evolutionary algorithms: A survey of the state of the art. Swarm and Evolutionary Computation 1(1): 32-49.

16. Conn AR, Scheinberg K, Toint PL (1997) Recent progress in unconstrained nonlinear optimization without derivatives. Mathematical Programming 79(1-3): 397-414.

17. Leticia CC, Susana CE, Coello CA (2008) Solving engineering optimization problems with the simple constrained particle swarm optimizer. Informatica 32(3): 319-326.

18. Harish G (2014) Solving structural engineering design optimization problems using an artificial bee colony algorithm. Journal of Industrial and Management Optimization 10(3): 777-794.

19. Fei Y, Haiyang Yu, Xueshou J (2010) An improved constrained optimization genetic algorithm. In: Intelligent Computing and Intelligent Systems (ICIS), 2010 IEEE International Conference on, Xiamen, China, 2: 435-439.
20. Seok KL, Zong WG (2005) A new meta-heuristic algorithm for continuous engineering optimization: harmony search theory and practice. Computer Methods in Applied Mechanics and Engineering 194(36-38): 3902-3933.

21. DF Jones, SK Mirrazavi, MTamiz (2002) Multi-objective metaheuristics: An overview of the current state-of-the-art. European Journal of Operational Research 137(1): 1-9.

22. Sadollah A, Bahreininejad A, Eskandar H, Hamdi M (2013) Mine blast algorithm: A new population based algorithm for solving constrained engineering optimization problems. Applied Soft Computing 13(5): $2592-2612$

23. Liqun G, Dexuan Z, Yanfeng G, Wenjing J (2010) Solving pressure vessel design problems by an effective global harmony search algorithm. In Control and Decision Conference (CCDC), Proceedings of Chinese, Xuzhou, Chinese, pp.4031-4035.

24. Tsung-Jung H (2014) A bacterial gene recombination algorithm for solving constrained optimization problems. Applied Mathematics and Computation 231: 187-204.

25. Jacqueline M, Richard C (1999) Application of particle swarm to multi objective optimization. Technical report, Department of Computer Science and Software Engineering, Auburn University, Alabama, USA.

26. Afshar A, Bozorg O, Marino MA, Adams BJ (2007) Honey-bee mating optimization hbmo algorithm for optimal reservoir operation. Journal of the Franklin Institute 344(5): 452-462.

27. Ali RY (2009) A novel particle swarm optimization approach for product design and manufacturing. International Journal of Advanced Manufacturing Technology 40(5-6): 617-628.

28. Wenyin G, Zhihua C, Dingwen L (2014) Engineering optimization by means of an improved constrained differential evolution. Computer Methods in Applied Mechanics and Engineering 268: 884-904.

29. Yong W, Zixing C, Yuren Z, Zhun F (2009) Constrained optimization based on hybrid evolutionary algorithm and adaptive constrainthandling technique. Structural and Multidisciplinary Optimization 37(4): 395-413.

30. Xin-She Y, Gandomi AH (2012) Bat algorithm: a novel approach for global engineering optimization. Engineering Computations 29(5): 464-483.

31. Ricardo LB, Coello CA (2006) Cultured differential evolution for constrained optimization. Computer Methods in Applied Mechanics and Engineering 195(33): 4303-4322.

32. Alex B, Jonathan V, Chukwudi A (2007) A review of particle swarm optimization. part I: background and development. Natural Computing 6(4): 467-484.

33. Janez B, Saso G, Borko B, Marjan M, Viljem Z (2006) Self-Adapting Control Parameters in Differential Evolution: A Comparative Study on Numerical Benchmark Problems. IEEE Transactions on Evolutionary Computation 10(6): 646-657.

34. Qie H, Ling W (2007) A hybrid particle swarm optimization with a feasibility-based rule for constrained optimization. Applied Mathematics and Computation 186(2): 1407-1422.

35. Hui L, Zixing C, Yong W (2010) Hybridizing particle swarm optimization with di erential evolution for constrained numerical and engineering optimization. Applied Soft Computing 10(2): 629-640.

36. Mezura-Montes E, Coello C (2005) Useful infeasible solutions in engineering optimization with evolutionary algorithms. In MICAI 2005: Advances in Artificial Intelligence 652-662.

37. Zhang M, Wenjian L, Wang X (2008) Differential evolution with dynamic stochastic selection for constrained optimization. Information Sciences 178(15): 3043-3074. 


\section{Robotics \& Automation Engineering Journal}

38. Leandro SC, Viviana CM (2008) Use of chaotic sequences in a biologically inspired algorithm for engineering design optimization. Expert Systems with Applications 34(3): 1905-1913.

39. Erik C, Miguel C (2014) A new algorithm inspired in the behavior of the social-spider for constrained optimization. Expert Systems with Applications 41(2): 412-425.

40. Adil H, Nishant G, Shruti G, Divya G (2013) Firefly algorithm for unconstrained optimization. IOSR Journal of Computer Engineering 11(1): 75-78.

41. Kennedy J, Eberhart RC (1995) Particle swarm optimization. In International Conference on Neural Network IV, Proceedings of the IEEE, Perth, Australia, pp. 1942-1948.

42. Marco D (1999) Ant colony optimization: A new meta-heuristic. In Evolutionary Computation (CEC'99), Proceedings of the 1999 Congress, Washington, USA, pp. 1470-1477.

43. Hofmeyr SA, Forrest S (2000) Architecture for an artificial immune system. Evol Comput 8(4): 443-473.

44. Passino KM (2002) Biomimicry of bacterial foraging for distributed optimization and control. IEEE Control System Magazine 22(3): 52-67.
45. Dervis K, Bahriye B (2007) A powerful and efficient algorithm for numerical function optimization: artificial bee colony $\mathrm{ABC}$ algorithm. Journal of Global Optimization 39(3): 459-471.

46. Timothy CH, Christopher J S, Nathan G S, James MK (2008) Roach infestation optimization. In Swarm Intelligence Symposium SIS 2008, Proceedings of the IEEE 1-7, St Louis, USA.

47. Xin-She Y (2010) A new metaheuristic bat-inspired algorithm. In Nature Inspired Cooperative Strategies for Optimization NICSO 2010 , p. 65-74.

48. Xin-She Y, Suash D (2009) Cuckoo search via levy flights. In Nature \& Biologically Inspired Computing (NaBIC), Proceedings of the 2009 World Congress, Coimbatore, India, pp. 210-214.

49. Xin-She Y (2009) Firefly algorithms for multimodal optimization. In Stochastic algorithms: foundations and applications 169-178.

50. Tawfeeq MA (2012) Intelligent algorithm for optimum solutions based on the principles of bat sonar. International Journal of Computer Science and Information Security 10(10): 1-9.

\section{Your next submission with Juniper Publishers} will reach you the below assets

- Quality Editorial service

- Swift Peer Review

- Reprints availability

- E-prints Service

- Manuscript Podcast for convenient understanding

- Global attainment for your research

- Manuscript accessibility in different formats

( Pdf, E-pub, Full Text, Audio)

- Unceasing customer service

Track the below URL for one-step submission https://juniperpublishers.com/online-submission.php 